\title{
Circulating miR-103 and miR-720 as novel serum biomarkers for patients with colorectal cancer
}

\author{
RYOJI NONAKA ${ }^{1 *}$, YUICHIRO MIYAKE $^{1 *}$, TAISHI HATA ${ }^{1}$, YOSHINORI KAGAWA $^{2}$, \\ TAKESHI KATO $^{2}$, HIDEKI OSAWA ${ }^{1}$, JUNICHI NISHIMURA ${ }^{1}$, MASAKAZU IKENAGA ${ }^{3}$, \\ KOHEI MURATA ${ }^{4}$, MAMORU UEMURA ${ }^{1}$, DAISUKE OKUZAKI ${ }^{5}$, ICHIRO TAKEMASA ${ }^{1}$, \\ TSUNEKAZU MIZUSHIMA ${ }^{1}$, HIROFUMI YAMAMOTO $^{1}$, YUICHIRO DOKI $^{1}$ and MASAKI MORI $^{1}$ \\ ${ }^{1}$ Department of Surgery, Gastroenterological Surgery, Graduate School of Medicine, Osaka University, Osaka; \\ ${ }^{2}$ Department of Surgery, Kansai Rosai Hospital, Hyogo; ${ }^{3}$ Department of Surgery, Osaka Rosai Hospital, Osaka; \\ ${ }^{4}$ Department of Surgery, Suita Municipal Hospital, Osaka; ${ }^{5}$ Special Research Facilities DNA-chip Development \\ Center for Infectious Diseases, Research Institute for Microbial Disease, Osaka University, Osaka, Japan
}

Received February 15, 2015; Accepted March 30, 2015

DOI: 10.3892/ijo.2015.3064

\begin{abstract}
Circulating microRNAs (miRNAs) have been reported as a biomarker for human malignancies, including colorectal cancer (CRC). The purpose of this study was to identify a novel biomarker for CRC through examination of serum miRNAs from the patients with CRC. Microarray analysis of miRNA expression was performed using paired pre- and post-operative serum from 10 CRC patients. miR-103 and miR-720 decreased significantly in the post-operative serum when compared to pre-operative serum. With an extended scale validation by qRT-PCR (quantitative real-time polymerase chain reaction) in $30 \mathrm{CRC}$ patients, we confirmed that serum miR-103 and miR-720 decreased significantly after surgery $(\mathrm{P}=0.0004$, and $\mathrm{P}=0.0274$, respectively). Next, we examined serum miR-103 and miR-720 levels in 32 non-cancer patients and $84 \mathrm{CRC}$ patients, and we found that expression of these two miRNAs was significantly higher in CRC patients than non-cancer patients. Furthermore, clinical and pathological survey indicated that high expression of miR-103 was significantly associated with histological differentiation grade, and lymphatic invasion and high expression of miR-720 was
\end{abstract}

Correspondence to: Dr Hirofumi Yamamoto, Department of Surgery, Gastroenterological Surgery, Graduate School of Medicine, Osaka University, 2-2 Yamada-oka, Suita City, Osaka 565-0871, Japan

E-mail: hyamamoto@gesurg.med.osaka-u.ac.jp

*Contributed equally

Abbreviations: CRC, colorectal cancer; miR, miRNA, microRNA; qRT-PCR, quantitative reverse transcription-polymerase chain reaction

Key words: miR-103, miR-720, colorectal cancer significantly associated with male gender and lymph node metastasis. Our data suggest that circulating miR-103 and miR-720 show potential as novel serum biomarkers for CRC.

\section{Introduction}

With the recent advances in chemotherapy, the prognosis for patients with metastatic colorectal cancer (CRC) has been considerably improved (1). However, CRC is still one of the most common malignancies worldwide and is a major cause of cancer-related deaths (2). Prognosis of this disease depends on tumor stage. The 5-year overall survival rates range from $93 \%$ for stage I patients to $8 \%$ for stage IV patients (3). Thus, early detection of CRC is important to reduce the mortality of this disease.

Representative tumor markers for CRC, carcinoembryonic antigen (CEA) and carbohydrate antigen (CA) 19-9 are not reliable in early detection of CRC. A previous study showed that serum p53 antibody test was a more sensitive tumor marker to detect early stage of CRC rather than CEA and CA19-9 (4). However, its feasibility as a screening test remained to be explored. Fecal occult blood test (FOBT) is employed as a screening test to detect CRC, but only $50-60 \%$ of early CRC is positive for FOBT. Currently, CRC is primarily diagnosed through colonoscopy. However, this procedure is invasive and expensive, and additionally requires bowel preparation, and may be associated with medical complications. Therefore, an optimal screening test that is easy to perform, noninvasive, acceptable, and can select those who have neoplastic lesions is required to improve the detection of CRC.

MicroRNAs (miRNAs) are newly discovered class of 22 nucleotide noncoding RNA molecules that regulate the translation and stability of specific target mRNAs through base-printing to partially complementary sites on target mRNAs that usually reside within the 3 ' untranslated regions (5). miRNAs have been considered to play an important role in the multistep processes of carcinogenesis either by oncogenic or tumor suppressive function (6). The association 
with tumorigenesis indicates their potential as diagnostic markers (7).

Circulating miRNAs are stably detected in plasma or serum and serve as biomarkers for several diseases, making them potentially useful noninvasive markers for early diagnosis or in monitoring of cancer progression $(8,9)$. Previous studies revealed that miRNAs were potential diagnostic or prognostic tools for CRC. For example, miR-92 or miR-21 is significantly elevated in plasma of CRC patients, and can be a potential noninvasive molecular marker for CRC detection $(10,11)$. We have recently found that serum miR-199a-3p is significantly higher in CRC patients than non-cancer patients, suggesting that circulating miR-199a-3p could be a biomarker for CRC (12). In this study, we explored other novel circulating miRNAs in CRC patients and evaluated its feasibility as a noninvasive diagnostic test for efficient detection of CRC.

\section{Materials and methods}

Patients and samples. Informed consent was obtained from CRC patients and non-cancer patients for the use of their blood samples. Venous blood samples were collected from the CRC patients $(n=114)$ and non-cancer patients $(n=32)$ from April 2011 to June 2013. In 30 of the CRC patients, blood samples were taken before and 7 th day after surgery. No cancer patients received chemotherapy or radiotherapy before blood sampling. The blood samples were obtained from Osaka University, and its associated hospitals. Whole blood was collected, centrifuged at $1,000 \mathrm{rpm}$ at $4^{\circ} \mathrm{C}$ for $15 \mathrm{~min}$. The supernatant fluids were centrifuged at $15,000 \mathrm{rpm}$ at $4^{\circ} \mathrm{C}$ for $10 \mathrm{~min}$. The supernatant fluids were stored at $-80^{\circ} \mathrm{C}$ until RNA extraction. This study was conducted under the supervision of the ethics board of Osaka University Hospital.

RNA extraction. Small RNA was enriched from all serum samples using the mirVana PARIS RNA isolation kit (Ambion, Austin, TX, USA), following the manufacturer's instructions. Briefly, $400 \mu \mathrm{l}$ of serum was thawed on ice and centrifuged at $15,000 \mathrm{rpm}$ for $15 \mathrm{~min}$ to remove cell debris. Next, $300 \mu \mathrm{l}$ of supernatant was lysed with an equal volume of $2 \mathrm{X}$ denaturing solution. For normalization of sample-to-sample variation during the RNA isolation procedures, 20 fmol of synthetic $C$. elegans miRNA cel-miR-39 was added to each denatured sample. Small RNAs were then enriched and purified following manufacturer's protocol. The concentration of all RNA samples were quantified by Nano Drop ND-1000 (NanoDrop Technologies, Wilmington, DE, USA).

miRNA microarray analysis. miRNA microarray experiments were carried out by using Agilent human miRNA microarray cataloged in the Sanger database version 12.0 (design ID 021827). About ten nanogram aliquots of total RNA with cel-miR-39 was used for making miRNA probes according to the Agilent protocol (version 2.3). Microarrays were performed for paired pre- and post-operative serum from 10 CRC patients. Briefly, total RNA was dephosphorylated with calf intestine alkaline phosphatase, denatured with dimethyl sulfoxide, and labeled with pCp-Cy3 using T4 RNA ligase using the miRNA Labeling Reagent and Hybridization kit. Probes were hybridized at $55^{\circ} \mathrm{C}$ for $20 \mathrm{~h}$ with rotation. Then the slides were washed by Gene Expression Wash Buffer 1 at room temperature for $5 \mathrm{~min}$ and by Gene Expression Wash Buffer 2 at $37^{\circ} \mathrm{C}$ for $5 \mathrm{~min}$. After hybridization and washing, the slides were scanned using an Agilent scanner (G2505C). Images were extracted using Agilent Feature Extraction software (version 10.7.3.1) and Agilent GeneSpring GX software (version 10.0.2). Differences in miRNA expression between the 10 pairs was determined if the fold change of cel-miR-39 normalized expression values was $>2.0$ and the P-value was $<0.05$ using paired t-test for further analysis. The microarray raw data are available in Gene Expression Omnibus (GEO;http://www.ncbi.nlm.nih.gov/geo) under accession number GSE55139.

$q R T-P C R$. For microRNA based RT-PCR assays, $2.5 \mu 1$ of enriched small RNAs from serum samples were reverse transcribed using the TaqMan MicroRNA Reverse Transcription kit (Applied Biosystems, San Diego, CA, USA) according to manufacturer's instructions in a total reaction volume of $7.5 \mu \mathrm{l}$. A 1:20 dilution of RT products was used as template for the PCR stage. PCR reaction was performed in triplicates using TaqMan 2X Universal PCR Master Mix according to the manufacturer's instructions. Each reaction was performed in a final column of $20 \mu \mathrm{l}$ containing $1.33 \mu \mathrm{l}$ of the cDNA and $1 \mu \mathrm{l}$ of Taqman miRNA assay mix. The amplification profile was: denaturation at $95^{\circ} \mathrm{C}$ for $10 \mathrm{~min}$, followed by 40 cycles of $95^{\circ} \mathrm{C}$ for $15 \mathrm{sec}$ and $60^{\circ} \mathrm{C}$ for $60 \mathrm{sec}$. Each sample was run in triplicates for analysis. The cycle threshold $(\mathrm{Ct})$ is defined as the number of cycles required for the fluorescent signal to cross the threshold in qPCR. The 7900 Sequence Detection System 2.3 (Applied Biosystems) software was used to compute the relative change in RNA expression by the $2-\Delta \Delta \mathrm{Ct}$ method with $95 \%$ confidence intervals.

Primers. The miRNA-specific primer sequences, including miRNA-103, miR-720, cel-miR-39, and RNU6B were designed based on the miRNA sequences obtained from the miRBase. The primer sequences are: hsa-miR-103, 5'-AGCAGCAUUG UACAGGGCUAUGA-3'; hsa-miR-720, 5'-UCUCGCUGGG GCCUCCA-3'; hsa-miR-21, 5'-UAGCUUAUCAGACUGAUG UUGA-3'; cel-miR-39, 5'-UCACCGGGUGUAAAUCAGC UUG-3'; RNU6B, 5'-CGCAAGGATGACACGCAAATTCG TGAAGCGTTCCATATTTTT-3'.

Statistical analysis. The significance of serum miRNA level was determined by Mann-Whitney test, Wilcoxon test and $\chi^{2}$ test where appropriate using the Graph Pad Prism 6 (San Diego, CA, USA). The sensitivity, specificity, and accuracy were calculated according to the standard formulas. Receiver operating characteristic (ROC) curves and area under the ROC curve (AUC) were established for discriminating patients with CRC. P-value of $<0.05$ was considered statistically significant.

\section{Results}

Results of miRNA microarray analysis. In comparison of CRC patient serum between pre- and post-operation $(n=10$; 4 stage II CRCs and 6 stage III CRCs) by the miRNA array, we identified miRNAs, most of which showed a decrease in 


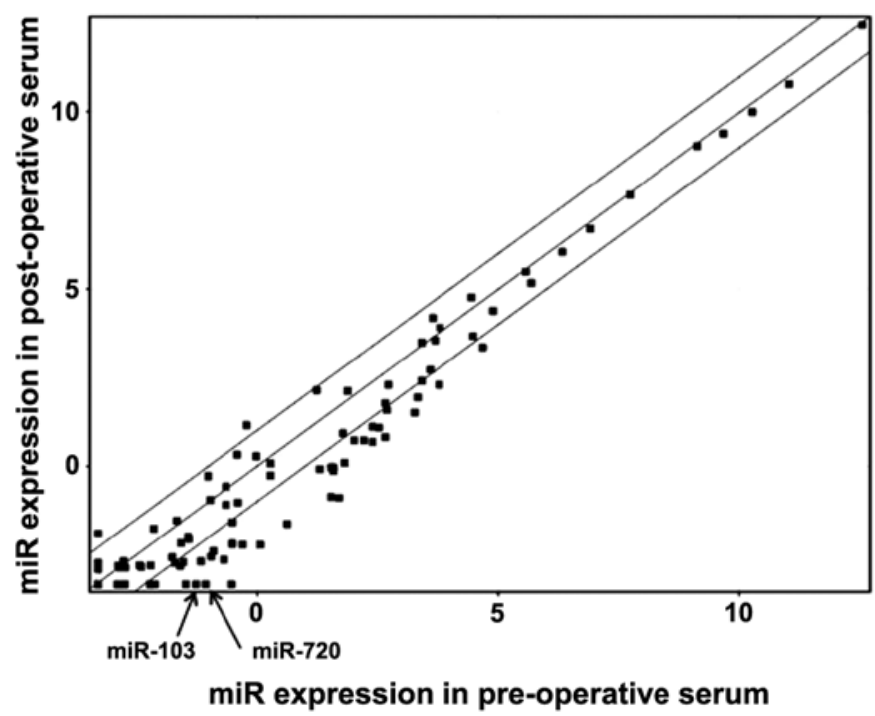

Figure 1. Results of miRNA array analysis. By comparison of patient serum between pre- and post-operation $(n=10)$ by miR array, two miRNAs (miR103, miR720) decreased in the post-operative serum as compared to the pre-operative serum $(\mathrm{P}=0.037$ and $\mathrm{P}=0.037$, respectively). The arrow and circle indicate miR-103 and miR-720.

the post-operative serum (Fig. 1). Among them, we focused on the two miRNAs, miR-103 and miR-720 whose expression showed a large decrease after surgery with significant $\mathrm{P}$-value (4.09-fold and 4.68-fold decrease; $\mathrm{P}=0.037$ and $\mathrm{P}=0.037$, respectively, Fig. 1).

Confirmation of results obtained by miRNA array by $q R T-P C R$. We then attempted to confirm the results obtained by miRNA array by qRT-PCR in extended samples of CRC patients $(n=30)$. As shown in Fig. 2, significant decrease in miRNA levels was noted in the post-operative serum for both miRNAs; $\mathrm{P}=0.0004$ for miR-103, and $\mathrm{P}=0.027$ for miR-720.
Expression of miRNAs in serum of normal and CRC patients. We examined serum miR-103 and miR-720 levels in 32 noncancer patients and 84 CRC patients. As shown in Fig. 3, both miRNAs were significantly higher in CRC patients than non-cancer patients $(\mathrm{P}=0.0067$ for $\mathrm{miR}-103$, and $\mathrm{P}=0.030$ for miR-720, respectively). ROC curve was drawn for serum miR-103 and miR-720, which yielded 0.662 for miR-103 and 0.630 for miR-720 as a value of AUC (Fig. 3). In discriminating CRC from non-tumor control subjects, the sensitivity and specificity of miR-103 were $55.9 \%$ and $75.0 \%$ at a cut-off point of 0.00081 ; these values for miR-720 were $58.3 \%$ and $56.3 \%$ at a cut-off point of 0.0016 .

Serum miR-21 expression in non-cancer and CRC patients. As a reference we examined miR-21 as a putative circulating miRNA. Using the same serum sets of normal and CRC patients, we found that serum miR-21 levels increased significantly in $\mathrm{CRC}$ patients as compared to non-tumor control patients $(\mathrm{P}=0.003)$. AUC of the ROC curves was 0.675 . When a cut-off point was set at 0.0107 , the sensitivity was $54.7 \%$ and the specificity was $84.4 \%$ in discriminating CRC from nontumor control subjects (Fig. 4).

Expression of miR-103 and miR-720 in patients serum and clinicopathological characteristics. CRC patients were divided into two groups by a median expression. Clinical and pathological survey indicated that high expression of miR-103 was significantly associated with poor differentiation and lymphatic invasion as compared to low miR-103 expression group $(\mathrm{P}=0.044$ and $\mathrm{P}=0.040$, respectively; Table I). High expression of miR-720 was significantly associated with male gender and lymph node metastasis as compared to low miR-720 expression group $(\mathrm{P}=0.001$ and $\mathrm{P}=0.048$, respectively; Table I).

Expression of miR-103 and miR-720 in normal mucosa and CRC tissue samples. We examined miR-103 and miR-720
$\operatorname{miR103}$

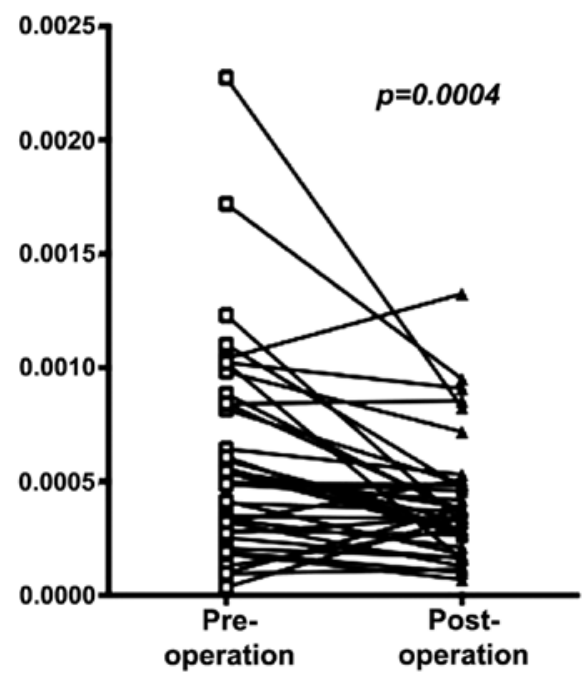

\section{$\operatorname{miR720}$}

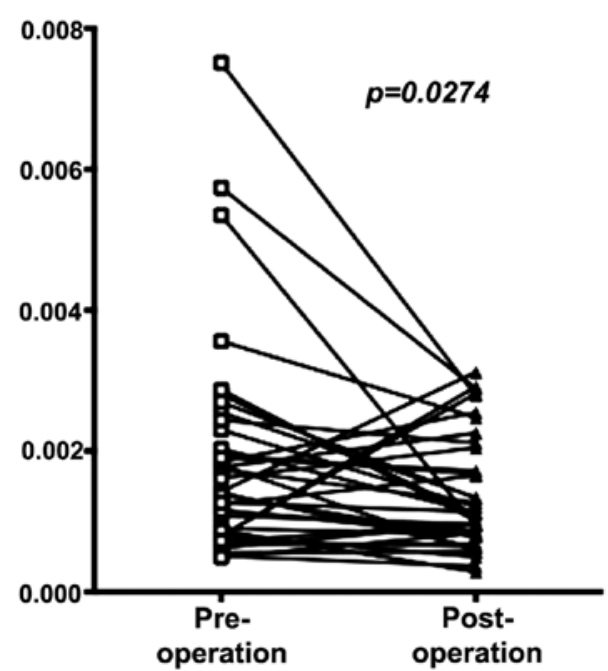

Figure 2. Confirmation of results obtained by miRNA array by qRT-PCR. We confirmed the results obtained by miRNA array by qRT-PCR in serum samples of CRC patients $(\mathrm{n}=30)$. A significant decrease in miRNA levels was noted in the post-operative serum when compared with pre-operative serum for both miRNAs; $\mathrm{P}=0.0004$ for miR-103, and $\mathrm{P}=0.027$ for miR-720. 
A

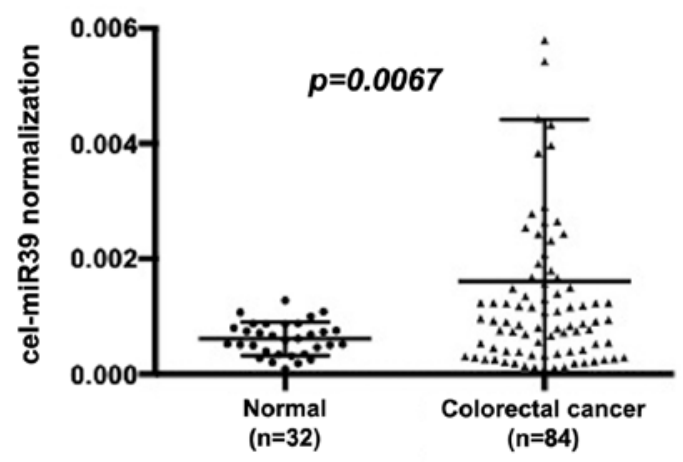

B

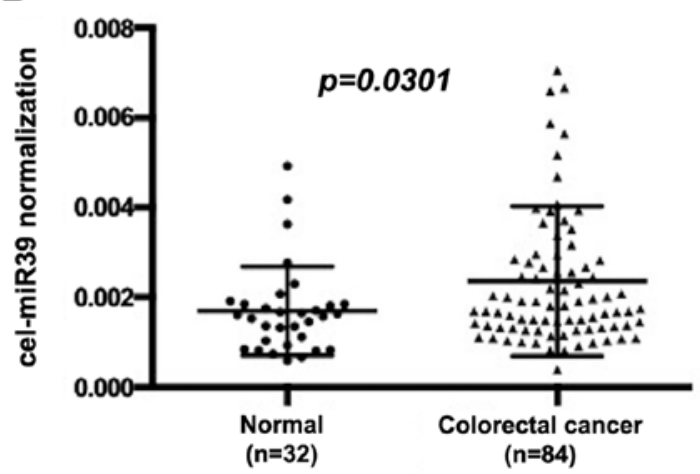

C
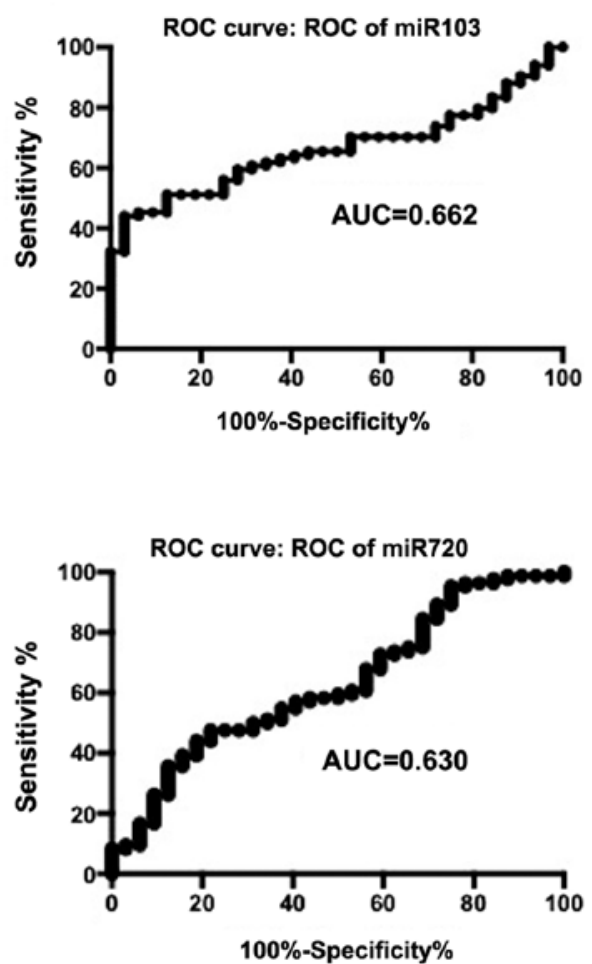

Figure 3. Comparison of serum miRNAs in non-tumor $(n=32)$ and CRC patients $(n=84)$. (A) Serum miR-103 expression in non-tumor and CRC patients. A significant increase in CRC patients was noted $(\mathrm{P}=0.0067)$. (B) Serum miR-720 expression in non-tumor and CRC patients. A significant increase in CRC patients was noted $(\mathrm{P}=0.030)$. (C) ROC curve was drawn for serum miR-103 and miR-720, which yielded 0.662 for miR-103 and 0.630 for miR-720 as a value of AUC. In discriminating CRC from non-tumor control subjects, the sensitivity and specificity of miR-103 were 55.9\% and $75.0 \%$ at a cut-off point of 0.00081 ; these values for miR-720 were $58.3 \%$ and $56.3 \%$ at a cut-off point of 0.0016 .

miR-21

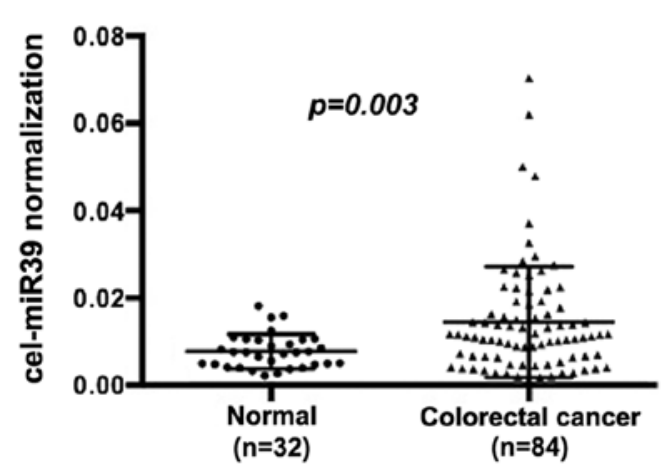

ROC curve of miR-21

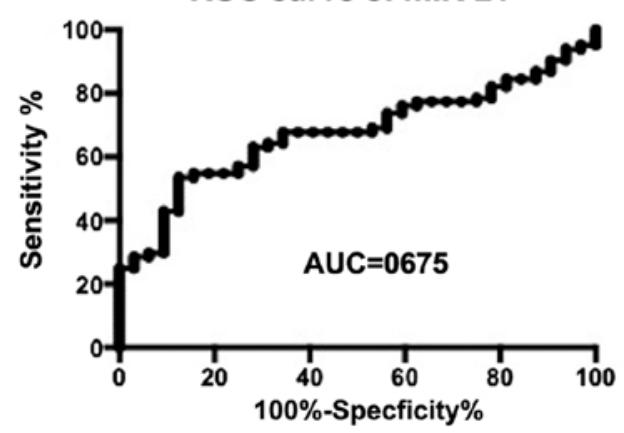

Figure 4. Serum miR-21 expression in non-cancer and CRC patients. Using the same serum sets of normal and CRC patients, we found that serum miR-21 levels increased significantly in CRC patients as compared to non-tumor control patients $(\mathrm{P}=0.003)$. AUC of the ROC curves was 0.675 . When a cut-off point was set at 0.0107 , the sensitivity was $54.7 \%$ and the specificity was $84.4 \%$ in discriminating CRC from non-tumor control subjects.

expression in normal colonic mucosa $(n=10)$ and CRC tissue samples $(\mathrm{n}=10)$. Both miRs were significantly lower in CRC tissue samples than in normal colonic mucosa $(\mathrm{P}=0.034$ for miR-103, and $\mathrm{P}=0.0052$ for miR-720) (Fig. 5).

\section{Discussion}

In this study, we explored to identify a novel serum marker for CRC by comparison of patient serum before and after operation, using microarray analysis of miRNAs. miRNAs were mostly reduced after surgery to various degrees (Fig. 1). Among them, miR-103 and miR-720 showed relatively large reduction after surgery. We validated the results by qRT-PCR in extended number of CRC patients (Fig. 2). These findings suggest that the two miRNAs could be derived from main CRC tumors, either directly or indirectly and that they may be useful to monitor the disease progression.

We demonstrated that the serum levels of miR-103 and miR-720 increased in CRC patients compared with non-cancer patients (Fig. 3). The ROC curves of miR-103 and miR-720 to 
Table I. Relation between miR-103, miR-720 and clinicopathological features.

\begin{tabular}{|c|c|c|c|c|c|c|}
\hline \multirow[b]{2}{*}{ Factors } & \multicolumn{3}{|c|}{ miR-103 } & \multicolumn{3}{|c|}{ miR-720 } \\
\hline & High & Low & P-value & High & Low & P-value \\
\hline Gender & & & 0.165 & & & $0.001^{\mathrm{a}}$ \\
\hline Male & 31 & 25 & & 35 & 21 & \\
\hline Female & 11 & 17 & & 7 & 21 & \\
\hline Differentiation & & & $0.044^{\mathrm{a}}$ & & & 0.503 \\
\hline tub1 & 12 & 21 & & 15 & 18 & \\
\hline tub2 por muc & 30 & 21 & & 27 & 24 & \\
\hline Tumor size & & & 1.000 & & & 1.000 \\
\hline$\geq 35 \mathrm{~mm}$ & 22 & 22 & & 22 & 22 & \\
\hline$<35 \mathrm{~mm}$ & 20 & 20 & & 20 & 20 & \\
\hline Serosal invasion & & & 0.172 & & & 1.000 \\
\hline Positive & 30 & 24 & & 15 & 27 & \\
\hline Negative & 12 & 18 & & 15 & 27 & \\
\hline $\begin{array}{l}\text { Lymph node } \\
\text { metastatis }\end{array}$ & & & 0.820 & & & $0.048^{\mathrm{a}}$ \\
\hline Positive & 19 & 18 & & 23 & 14 & \\
\hline Negative & 23 & 24 & & 19 & 28 & \\
\hline Lymphatic invasion & & & $0.040^{\mathrm{a}}$ & & & 0.827 \\
\hline Positive & 25 & 18 & & 21 & 22 & \\
\hline Negative & 13 & 24 & & 21 & 20 & \\
\hline Venous invasion & & & 0.474 & & & 0.095 \\
\hline Positive & 14 & 11 & & 16 & 9 & \\
\hline Negative & 28 & 31 & & 26 & 33 & \\
\hline
\end{tabular}

Well, well differentiated adenocarcinoma; mod, moderately differentiated adenocarcinoma; por, poorly differentiated adenocarcinoma;

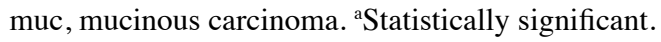

distinguish cancer patients from non-cancer patients appeared to have limitation in sensitivity and specificity. To estimate its value, we examined serum miR-21 in the same series of non-tumor and CRC patients. Serum miR-21 is well known as a serum biomarker for CRC (13). We found that diagnostic power of miR-103 was similar to that of miR-21 in our series but that miR-720 showed limited specificity as compared to miR-21. Thus, miR-103 might be better biomarker for CRC than miR-720.

Clinicopathological survey showed that high expression of serum miR-103 was associated with poor differentiation and lymphatic invasion. Chen et al showed that, miR-103 promoted metastasis of CRC by targeting the metastasis suppressors DAPK and KLF4. This study also showed that miR-103 had a role in downregulating E-cadherin, claudin-3, and occludin, which sensitized tumor cells to EMT-inducing signals, and led to local invasion (14). Additionally, we found that high expression of serum miR-720 was associated with lymph node metastasis. The finding is consistent with a report by Wang et al that high level of miR-720 in CRC tissues
miR-103

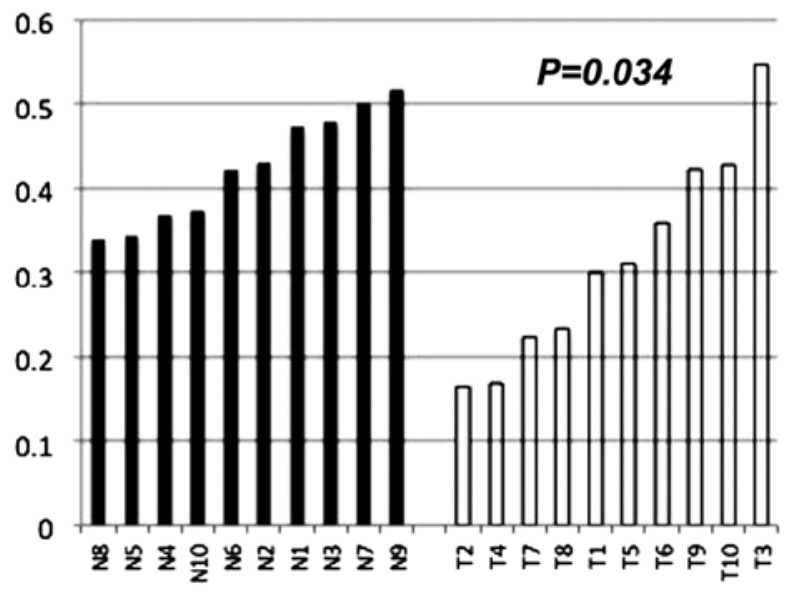

miR-720

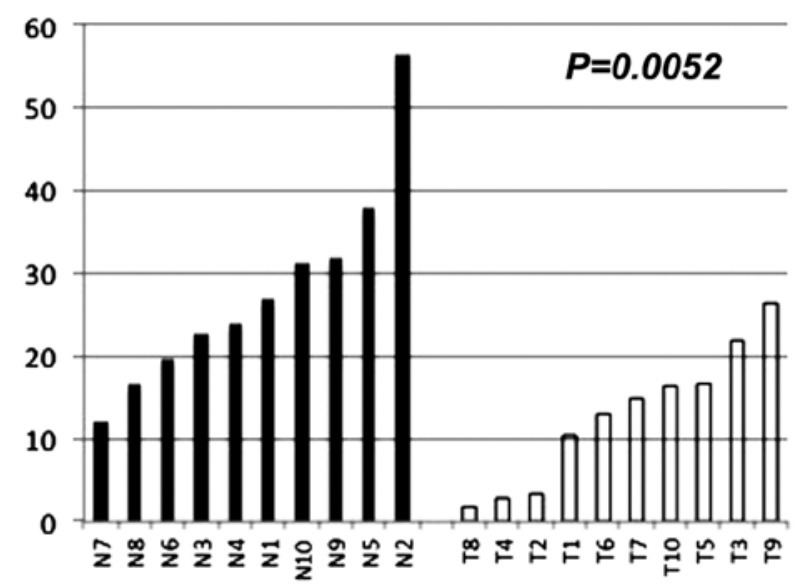

Figure 5. Expression of miR-103 and miR-720 in normal mucosa and CRC tissue samples. We additionally examined miR-103 and miR720 expression in normal colonic mucosa $(n=10)$ and CRC tissue samples $(n=10)$. Both miRs were significantly lower in CRC tissue samples than in normal colonic mucosa $(\mathrm{P}=0.034$ for miR-103, and $\mathrm{P}=0.0052$ for $\mathrm{miR}-720)$.

correlated with the tumor size, lymphatic metastasis, distant metastasis, and poor prognosis (15).

In analysis of miR-103 and miR-720 derived from the tissue samples, we found that the expression levels of the two miRNAs, especially miR-720 were significantly lower in CRC tissue samples than in normal colonic mucosa (Fig. 5). The contradictory miRNA expression levels between the tumor tissues and the serum have been demonstrated as to several miRNAs, but this phenomenon remains unexplained and needs to be further explored $(16,17)$. A previous in vitro study showed that miRNA profiles in a conditioned medium were different from those in cells, and it is thus suggested that the secreted miRNAs represent a class of signaling molecules in mediating intercellular communication (18). The precise mechanism of miRNA packaging and secretion is largely unknown, and investigation of the mechanism is a future issue of miRNA research (19).

In conclusion, we identified miR-103 and miR-720 from differential expression profile between pre- and postoperation as novel serum biomarkers for CRC. Further investigation of serum miR-103 and miR-720 in patient prog- 
nosis and monitoring therapeutic efficacy of chemotherapy is essential.

\section{Acknowledgements}

This study was supported by a Grant-in-Aid for Scientific Research (B) (24390315 to H.Y.).

\section{References}

1. Inoue $\mathrm{Y}$ and Kusunoki M: Advances and directions in chemotherapy using implantable port systems for colorectal cancer: A historical review. Surg Today 44: 1406-1414, 2014.

2. Jemal A, Siegel R, Ward E, Hao Y, Xu J and Thun MJ: Cancer statistics, 2009. CA Cancer J Clin 59: 225-249, 2009.

3. Hewitson P, Glasziou P, Watson E, Towler B and Irwig L: Cochrane systematic review of colorectal cancer screening using the fecal occult blood test (hemoccult): An update. Am J Gastroenterol 103: 1541-1549, 2008.

4. Ochiai H, Ohishi T, Osumi K, Tokuyama J, Urakami H, Seki S, Shimada A, Matsui A, Isobe Y, Murata Y, et al: Reevaluation of serum p53 antibody as a tumor marker in colorectal cancer patients. Surg Today 42: 164-168, 2012.

5. Mendell JT and Olson EN: MicroRNAs in stress signaling and human disease. Cell 148: 1172-1187, 2012.

6. Schetter AJ, Leung SY, Sohn JJ, Zanetti KA, Bowman ED, Yanaihara N, Yuen ST, Chan TL, Kwong DL, Au GK, et al: MicroRNA expression profiles associated with prognosis and therapeutic outcome in colon adenocarcinoma. JAMA 299: 425-436, 2008

7. Lu J, Getz G, Miska EA, Alvarez-Saavedra E, Lamb J, Peck D, Sweet-Cordero A, Ebert BL, Mak RH, Ferrando AA, et al MicroRNA expression profiles classify human cancers. Nature 435: 834-838, 2005.

8. Chen X, Ba Y, Ma L, Cai X, Yin Y, Wang K, Guo J, Zhang Y, Chen J, Guo X, et al: Characterization of microRNAs in serum: A novel class of biomarkers for diagnosis of cancer and other diseases. Cell Res 18: 997-1006, 2008.
9. Fabbri M: miRNAs as molecular biomarkers of cancer. Expert Rev Mol Diagn 10: 435-444, 2010.

10. Ng EK, Chong WW, Jin H, Lam EK, Shin VY, Yu J, Poon TC, $\mathrm{Ng}$ SS and Sung JJ: Differential expression of microRNAs in plasma of patients with colorectal cancer: A potential marker for colorectal cancer screening. Gut 58: 1375-1381, 2009.

11. Kanaan Z, Rai SN, Eichenberger MR, Roberts H, Keskey B, Pan J and Galandiuk S: Plasma miR-21: A potential diagnostic marker of colorectal cancer. Ann Surg 256: 544-551, 2012.

12. Nonaka R, Nishimura J, Kagawa Y, Osawa H, Hasegawa J, Murata K, Okamura S, Ota H, Uemura M, Hata T, et al: Circulating miR-199a-3p as a novel serum biomarker for colorectal cancer. Oncol Rep 32: 2354-2358, 2014.

13. Toiyama Y, Takahashi M, Hur K, Nagasaka T, Tanaka K, Inoue Y, Kusunoki M, Boland CR and Goel A: Serum miR-21 as a diagnostic and prognostic biomarker in colorectal cancer. J Natl Cancer Inst 105: 849-859, 2013.

14. Chen HY, Lin YM, Chung HC, Lang YD, Lin CJ, Huang J, Wang WC, Lin FM, Chen Z, Huang HD, et al: miR-103/107 promote metastasis of colorectal cancer by targeting the metastasis suppressors DAPK and KLF4. Cancer Res 72: 3631-3641, 2012.

15. Wang X, Kuang Y, Shen X, Zhou H, Chen Y, Han Y, Yuan B, Zhou J, Zhao H, Zhi Q and Xue X: Evaluation of miR-720 prognostic significance in patients with colorectal cancer. Tumour Biol 36: 719-727, 2015.

16. Yang IP, Tsai HL, Huang CW, Huang MY, Hou MF, Juo SH and Wang JY: The functional significance of microRNA-29c in patients with colorectal cancer: A potential circulating biomarker for predicting early relapse. PLoS One 8: e66842, 2013.

17. Heegaard NH, Schetter AJ, Welsh JA, Yoneda M, Bowman ED and Harris CC: Circulating micro-RNA expression profiles in early stage nonsmall cell lung cancer. Int J Cancer 130: 1378-1386, 2012.

18. Zhang Y, Liu D, Chen X, Li J, Li L, Bian Z, Sun F, Lu J, Yin Y, Cai X, et al: Secreted monocytic miR-150 enhances targeted endothelial cell migration. Mol Cell 39: 133-144, 2010.

19. Cheng H, Zhang L, Cogdell DE, Zheng H, Schetter AJ, Nykter M, Harris CC, Chen K, Hamilton SR and Zhang W: Circulating plasma MiR-141 is a novel biomarker for metastatic colon cancer and predicts poor prognosis. PLoS One 6: e17745, 2011. 